\title{
Epistemic Peer Disagreement
}

\author{
Filippo Ferrari (University of Bonn) \\ Nikolaj Jang Lee Linding Pedersen (Yonsei University) ${ }^{1}$
}

Word-count: 5,406

\section{The epistemic significance of perceived peer disagreement}

Recently the following issue has been widely discussed by epistemologists:

(Significance)

What is the epistemically rational response in the face of mutually recognized disagreement?

(Significance) pertains to what attitude an agent is epistemically rational in holding in light of disagreement.

Why should we think that disagreement is of philosophical significance? One reason is that disagreement functions as a sort of litmus paper for detecting the presence of error (Sidgwick 1907: 342, Christensen 2007: 8). The exact nature and significance of this error need to be scrutinized. To that end, we introduce three examples of disagreement about different subject matters: ordinary perception, arithmetic, and economic policy.

(Perception) - You and I are traffic cops watching cars pass on Main Street. We are equally good, equally attentive cops, with equally reliable eyesight and regard each other as such. We see a truck pass through the intersection. I think that it ran the red light. You think it got through on yellow. (Vavova 2014: 307)

(Arithmetic) - You and I add a series of ten three digit numbers in our heads. A third party calls out the numbers, one after the other. Each of us keeps a tally, adding the numbers as we go, not attempting to keep track of any particular number in the sequence after it has been added to the running total. Neither I nor you are particularly good at mental arithmetic. We both recognize this and that, when we have played this game in the past, we have made a more or less equal number of mistakes. This time I arrive at the number 5,863 but you think that this is not the right answer. 
(Economic policy) - Alberto and Paul are both leading economists who recognize each other as such. They have been engaged in a long debate about economic policy in Europe, exchanging arguments and considerations over an extended period of time. Alberto claims that spending cuts are associated with economic expansions and this is because confidenceinspiring policies will foster and not hamper economic recovery in Europe. Paul, on the other hand, maintains that this confidence leads to disastrous results and that austerity is a self-defeating policy which does great harm to European economies, making their debt grow even faster.

When subjects take each other to be epistemically on a par, they are said to regard each other as epistemic peers. The idea of epistemic peerhood can be cashed out as follows (Christensen 2007 and 2008, Feldman 2006 and 2007, Gutting 1982, Kelly 2005, Lackey 2010a and 2010b):

(Epistemic peers)

$S_{1}$ and $S_{2}$ are epistemic peers regarding $p$ if and only if

(i) $\quad S_{1}$ and $S_{2}$ are cognitive equals, i.e. they are equals with respect to cognitive virtues such as intelligence, thoughtfulness, competence, and freedom from bias, and

(ii) $\quad S_{1}$ and $S_{2}$ are evidential equals, i.e. they are equally familiar with the evidence and arguments that concern $p$ and have considered them with equal care.

The first condition pertains to the general cognitive profile of subjects. This impacts how they engage in enquiry-how they deliberate, reason, and how they search for, select, and process evidence. The second condition concerns the relative standing of the subjects vis-a-vis evidence pertaining specifically to the question whether $p$.

The three cases of disagreement just presented raise the following instance of (Significance):

(Significance-Peer)

What is the epistemically rational response in the face of mutually recognized disagreement between subjects who take each other to be epistemic peers? 
This is a difficult question. Some cases of disagreement involve a perceived asymmetry: one party takes the other to be epistemically superior, but the converse does not hold. This happens when someone recognized as an expert about a given subject matter disagrees with someone regarded as a novice. In such cases, the rational response would seem for the recognized expert to stick to her initial belief and for the novice to defer to her. However, the three cases just presented do not involve this kind of asymmetry and, thus, seem to be much harder to deal with. For this reason, recognized peer disagreement has been at the heart of the recent epistemological literature on disagreement. Different answers to (Significance-Peer) have been given. We present some prominent answers in the next two sections. $^{2}$

Before proceeding let us note that some epistemologists work within a degree-theoretic framework, representing degrees of beliefs by real number in the unit interval $(0 ; 1)$. Degrees of beliefs behave like subjective probabilities, indicating how likely a subject takes a given proposition to be. Other epistemologists work with three non-graded attitudes: belief, disbelief, and suspension of belief. Disbelieving $p$ is understood as believing not- $p$, and suspension about whether $p$ is understood as some sort of neutral attitude, contrasting with both believing and disbelieving $p$ (Friedman 2013). Within the degree-theoretic framework, disagreement occurs if two subjects have different degrees of belief. Thus, if Sarah's degree of belief that Paris is in France is 0.95 and Bob's degree of belief in the same proposition is 0.6, Sarah and Bob disagree. Within the framework of full attitudes two subjects disagree if one believes $p$ and the other disbelieves $p$. Both frameworks are widely used in the discussion of peer disagreement. For this reason we rely on both of them below.

\section{Conformism vs. non-conformism}


There are two main views on (Significance-Peer): conformism and non-conformism. Conformism is the following view:

(Conformism)

In light of mutually recognized peer disagreement regarding $p$ the epistemically rational response for both parties is to revise their doxastic attitude towards $p$ so it is closer to that of the other party.

Conformism predicts that disagreement with a recognized peer is always epistemically significant. Such disagreement rationally requires both parties to abandon their initial belief (non-degreetheoretic framework) or their initial credence (degree-theoretic framework) and move closer to the other party. Hence the label "conformism" (another commonly used label is "conciliationism").

The perhaps most widely discussed form of conformism is the equal weight view - thus called because it requires each view to carry equal weight:

(Conformism $\left.^{\text {ew-full }}\right)$

In light of mutually recognized peer disagreement regarding $p$ the epistemically rational response for both parties is to suspend belief regarding $p$ (Feldman 2006, 2007).

(Conformism $^{\text {ew-degree }}$ )

In light of mutually recognized peer disagreement regarding $p$ the epistemically rational response for both parties is to assign each view equal weight. Put mathematically: if $S_{I}$ 's credence in $p$ is $x$ and $S_{2}$ 's credence is $y$ (where $x \neq y$ ), the credence of both $S_{1}$ and $S_{2}$ should equal the average of $x$ and $y$ (Christensen 2007, 2008, Elga 2007).

Within a full belief framework, the two parties' views carry equal weight in the sense that their respective beliefs cancel each other out: they should both suspend belief about $p$. Within a degreetheoretic framework, recognized disagreement carries equal weight in the sense that the credence of both parties should equal the average of the two initial credences.

All versions of (Conformism) are symmetric in that they require doxastic revision from both parties. However, it is compatible with (Conformism) that the parties do not have to revise in the same way - that, e.g., one party should assign 0.8 weight to the other party's view and 0.2 to her 
own. Note, however, that equal weight versions of conformism are symmetric twice over: both parties are rationally required to doxastically revise, and they have to do so in the same way.

According to (Conformism ${ }^{\text {ew-full }}$ ), this means that both $S_{1}$ and $S_{2}$ should suspend belief about p. In (Perception) the disagreeing traffic cops should suspend belief about whether the car ran a red light. The same goes for (Arithmetic) and (Economic policy). According to (Conformism ${ }^{\text {ew-degree }}$ ) the disagreeing parties should "split the difference" in each case. Suppose that in all three cases the initial degrees of belief of the disagreeing parties are respectively 0.8 and 0.2 . Then the degree of belief of both traffic cops in $<$ The car ran a red light $>$ should be 0.5 . Similarly, in the arithmetical and the economic policy cases.

Non-conformism is the view that disagreement with a perceived peer does not always rationally mandate doxastic revision. This can happen symmetrically or asymmetrically:

(Non-conformism $^{\mathrm{SYM}}$ )

In light of mutually recognized peer disagreement regarding $p$ it can be epistemically rational for both parties to maintain their initial doxastic attitude towards $p$ (Foley 2001, Rosen 2001, Wedgwood 2007).

(Non-conformism ${ }^{\mathrm{ASYM}}$ )

In light of mutually recognized peer disagreement regarding $p$ it can be epistemically rational for one party (but not both) to maintain her initial doxastic attitude towards $p$ (Kelly 2005, Lackey 2010a, 2010b).

To get the full-attitude version of symmetric and asymmetric non-conformism replace "doxastic attitude" with "belief", to get the degree-theoretic version replace it with "credence".

How might (Non-conformism ${ }^{\mathrm{SYM}}$ ) be supported? Here is a simple argument: suppose that $S_{I}$ believes $p$. The truth of not- $p$ is incompatible with the truth of $p$. So, when $S_{1}$ learns of the disagreement with $S_{2}$, from $S_{1}$ 's perspective there is reason to think that $S_{2}$ is wrong. Similarly from the perspective of $S_{2}$. Thus, both $S_{1}$ and $S_{2}$ can rationally maintain their initial doxastic attitude. 
This line of argument embodies a form of "epistemic egocentrism", as it relies on the idea that the first-person perspective is privileged and epistemically decisive for each subject. ${ }^{3}$

How about (Non-conformism $\left.{ }^{\mathrm{ASYM}}\right)$ ? Some endorse this form of asymmetric nonconformism by appealing to the so-called "right reasons view" (RRV): in cases of disagreement between recognized peers the party who in fact reasoned correctly from the evidence can rationally maintain her initial doxastic attitude while the other party cannot. This former party has the right reasons supporting her doxastic attitude. E.g., in (Arithmetic) suppose that the sequence of numbers in fact adds up to 5,863, and that this is your claim. In that case you can rationally maintain your initial belief that the sum of the sequence is 5,863 while I should revise my conflicting belief.

\section{Variations}

Let us introduce three further views concerning recognized peer disagreement: the total evidence view, the justificationist view, and the reasoning as grounding view. These can be regarded as refinements of conformism or non-conformism.

According to the total evidence view (TEV) the body of total evidence determines what response is epistemically rational in the face of recognized peer disagreement. TEV includes both first-order evidence and higher-order evidence, respectively the evidence concerning $p$ considered by the disagreeing parties and information about the disagreement (e.g., the epistemic status of the other party (or parties) and how many people hold the different views; Kelly 2010, Sect. 4). When a subject's total evidence includes the information that a recognized peer holds a different view, TEV mandates doxastic revision: the subject should be less confident. So, TEV is a conformist view. However, it does not amount to equal weight conformism. The view says that some-but not necessarily equal — weight should be accorded to the view of a recognized peer.

Consider the following case: 
(Conjecture) - A mathematician proves a theorem that settles what has long been an open question - say, the Twin Prime Conjecture (i.e. the conjecture that there are infinitely many pairs of primes separated by a difference of two). However, suppose also that the whole mathematical community contests the proof (this case is modeled on Case 6 in Kelly 2010).

TEV predicts that the mathematician should be less confident although she has reasoned correctly and in fact proved the Twin Prime Conjecture. In this respect, TEV is different from the RRV, which would attribute no significance to other members of the mathematical community because they have reasoned incorrectly. However, while conformist in nature, TEV is different from the equal weight view. It is compatible with TEV-but not RRV-that the mathematician can rationally remain quite confident in her initial belief vis-à-vis the disagreement.

According to the justificationist view (JV), what counts as epistemically rational responses in the face of recognized peer disagreement is determined by the subjects' degree of justified confidence and, in some cases, certain kinds of information. If a subject has a low degree of justified confidence, then substantial doxastic revision is rationally required in the face of recognized peer disagreement. However, if she has a high degree of justified confidence, she can rationally maintain her initial doxastic attitude just in case she has a symmetry-breaker. A symmetry-breaker consists in personal information to the effect that her own epistemic situation is good together with the absence of this kind of information about the other party (Lackey 2010a, 2010b).

$\mathrm{JV}$ is a non-conformist view, as it allows for no-revision cases. To see this consider the following case:

(Pasta) - Jen is at the dining table with her flat mate Joe. Joe has a vivid experience as of talking to Sarah, their other flat mate, and as of her eating pasta with great gusto. Jen does not have this kind of experience-she has an experience as of Sarah's not being present. Joe says to Jen, "Wow! Sarah is really enjoying the pasta!" Jen responds, "Sarah isn't here." Jen and Joe regard each other as peers with respect to perceptual judgments and have the same kind of evidence, perceptual experience. Jen is justified in believing about herself that she is not drunk; is not under the influence of any drugs; has 20/20 vision; and that she has no 
history of hallucinating in the past. Furthermore, Jen does not have this kind of information about Joe. Jen is having veridical perceptual experiences.

Jen has a high degree of justified confidence that Sarah is not present on the basis of ordinary perception. She also has personal information that provides a symmetry-breaker. For this reason Jen can rationally maintain her initial belief that Sarah is not present (Lackey 2010a). This suffices to show that JV is a brand of non-conformism.

Note that JV delivers doxastic revision verdicts for some cases and provides diagnostics for identifying them. To see this consider (Arithmetic). Since the two subjects are not mathematically gifted, they both have a low degree of justified confidence. Thus, according to JV, when they learn of the disagreement with one another, they are both rationally required to substantially revise their initial doxastic attitude.

Wietmarschen (2013) draws a distinction between well-grounded belief and evidentially supported belief. One thing is to say that evidence $E$ supports $S$ 's belief that $p$-and thus gives $S$ justification to believe that $p$; another thing is to say that $S$ 's belief that $p$ is epistemically wellgrounded by $S$ 's evidence - and thus that $S$ is justified in believing that $p$. Wietmarschen defends the reasoning as grounding view (RGV) — a form of conformism, understood as a view about wellgrounded belief. The distinction between evidentially supported and epistemically well-grounded belief allows for the possibility that a belief that $p$ is epistemically supported by evidence $E$ without being well-grounded by $E$. By appealing to this distinction one can assuage some of the problematic consequences that other forms of conformism have - in particular, the concern that conformist views seem to make the original evidence on which your belief is based epistemically insignificant (or at least, much less significant than before) once you come to know about the disagreement with your peer (Kelly 2010: 122-25). 
To illustrate the mechanics of RGV return to (Conjecture). Initially the mathematician's belief that the Twin Prime Conjecture is true is well-grounded by her evidence, in the form of what she takes to be (and what is in fact) a proof of the conjecture. However, when she learns of the opposition from the mathematical community (including many mathematicians whom she regards as peers), this information provides a potential defeater. RGV predicts that this potential defeater undermines the well-groundedness of the mathematician's belief. Thus she is no longer justified in believing the Twin Prime Conjecture and should doxastically revise. However, she still has justification to believe the conjecture. This is because her evidence is, in fact, a proof of the conjecture and evidentially supports her belief.

\section{Assessing the views}

In this section, we critically assess some of the views outlined above. The current literature is rich with interesting objections and challenges. However, given limitations of space we confine ourselves to a cursory review of a few prominent criticisms of the views presented in Sect. 2 .

Let us first review the pros and cons of conformism. One general virtue of conformism is that it aligns well with the natural idea that disagreement with someone regarded as a peer provides evidence that one has made a mistake in interpreting the original evidence, and that such evidence should make one less confident. However, conformist proposals have been subject to a variety of criticisms. We will briefly review four of them.

The first criticism puts pressure on the commitment to treat as epistemically irrelevant one's original evidence, once the disagreement with a peer comes to light. This seems highly problematic because it completely disregards the possibility that one of the disagreeing parties might have reasoned impeccably, while the other has reasoned incorrectly (see Kelly 2010: 122-25 for the criticism and Christensen 2011: 4 for a reply). 
A second issue with conformism is its seeming self-defeating character. Suppose that as an epistemically well-informed conformist I disagree with an epistemically equally well-informed nonconformist about the epistemic significance of peer disagreement. By my own lights in such a situation I should conciliate by decreasing my confidence in the correctness of my own view (or, working with full attitudes, suspend belief). But this seems absurd since it implies that "your view on disagreement requires you to give up your view on disagreement” (Elga 2010: 179. See also Weatherson 2013 and Reining 2016).

A third worry about conformism takes issue with the so-called Independence Principle-a key commitment of the view. As Christensen (2009: 758) formulates it: "In evaluating the epistemic credentials of another person's belief about $p$, to determine how (if at all) to modify one's own belief about $p$, one should do so in a way that is independent of the reasoning behind one's own initial belief about $p$." Suppose that I am extremely confident not only about the truth of my belief that $p$ but also about the extreme reliability of the reasoning process that led me to believe that $p$. Let us suppose that I can fully introspect this process and assess its reliability in a way that, on this occasion, I have no reason to doubt is flawed. Moreover, suppose that I lack introspective awareness of the reliability of the other party's reasoning process on this occasion. It would seem that I have reason not to conciliate. It would also seem that I have reason to think that the other party has made a mistake on this particular occasion. However, my reasons for not conciliating and for attributing fault to my opponent are not independent of the reasoning behind my initial belief and reasoning about $p$. Thus, they would be ruled out as epistemically significant in a conformist framework that incorporates the Independence Principle (Sosa 2010 and Lackey 2010a; see Christensen 2011: 9 for a reply and Christensen 2016, Kelly 2013: 37-43, and Lord 2014 for a comprehensive discussion of the Independence Principle). 
A fourth worry concerns the so-called Uniqueness Thesis - the idea that there is a uniquely rational response in the face of disagreement with a perceived peer. Versions of conformism appear to be committed to this thesis. For example, the equal weight view seems to be committed to saying that suspension of belief (Feldman 2006, 2007) or adopting the average of the two initial degrees of belief (Christensen 2007, 2008, Elga 2007) is the uniquely rational response for both parties. Some worry about uniqueness due to specific cases of disagreement that they take to constitute counterexamples. For example, people who grow up in religious communities and people who grow up among atheists might rationally take the same arguments or body of evidence to support different conclusions (the existence/non-existence of God) due to influences from their different backgrounds (Schoenfield 2014; for a non-case-based argument see Ballantyne and Coffman 2011; for an overview see Kopec and Titelbaum 2016, for defences see Christensen 2016 and White 2005).

Let us now turn to a brief discussion of non-conformist views. One general advantage of such views is that they pay due respect to the subject's original evidence, gathered prior to the situation of disagreement. After all, the mere fact that we happen to disagree with a peer should not make all the relevant evidence available to us up to that point epistemically insignificant. Moreover, non-conformist views support our strong intuition that in many cases where we can have introspective access to the reliability of the reasoning process that led to our opinion on the disputed matter, the fact that it is our own opinion and reasoning process we are considering should make a difference. However, there are also reasons to be skeptical about the general tenability of nonconformist views.

One general worry is that a non-conformist attitude towards situations of recognized peer disagreement seems to encourage dogmatism leading, at its extreme, to the permissibility of a subject's systematically disregarding any unfavorable evidence or contrary opinion (see Pedersen 
2018 on strong forms of non-conformism). This seems to clash with the fact that we genuinely regard our opponent as an epistemic peer. And this, of course, would be a rather unwelcome consequence, especially in all those cases where neither subject has reason to believe that the situation is epistemically asymmetrical.

A related, but more specific, worry is discussed by Christensen (2007: 209; 2011: 5). Disagreement with your peer provides new evidence that significantly modifies your epistemic situation compared to your pre-disagreement situation. Non-conformist views seem to seriously downplay the epistemic significance of the presence of disagreement which is clearly a weakness of the view especially in those cases where both disputants regard each other as epistemic peers. After all, the fact that I regard you as an epistemic peer and that you disagree with me about whether $p$ is a significant piece of evidence that should be factored in in my current epistemic situationevidence that somehow challenges the good standing of my pre-disagreement epistemic situation.

What these very general criticisms of conformism and non-conformism suggest is that neither of them can be said to successfully provide a wholly general and adequate answer to (Significance-Peer). Non-conformist views seem right—contra conformist views-when they insist that the epistemic significance of our initial evidence should not be completely discarded in the face of disagreement with someone regarded as a peer. Conformists have it right-contra nonconformists - when they insist that the presence of disagreement with a perceived peer has epistemic significance and changes the epistemic situations of the disputants. The three alternative pictures reviewed in Sect. 4 can be seen, roughly, as alternative ways of accommodating the positive features of both conformism and non-conformism while avoiding their respective shortcomings. In this respect, such alternatives represent genuine progress in the debate on peer disagreement. 


\section{Disagreement and varieties of rationality}

We are inclined to think that the peer disagreement debate has been conducted somewhat in isolation from other debates in epistemology. This is unfortunate since the epistemic significance of peer disagreement very much seems to be an issue related to other issues in epistemology. Differences between views on peer disagreement may well reflect—or be traceable to — differences in terms of broader epistemological commitments. To argue this point we draw a distinction between two kinds of rationality and discuss them in relation to the views introduced earlier.

Let us distinguish between internal and external rationality. A belief's being internally rational amounts to its being (i) an appropriate response to phenomenal experiences associated with sensation or perception (being appeared to redly, etc.) or the exercise of memory, a priori reasoning, or introspection (seeming right, etc.), (ii) a member of a coherent belief system, (iii) a trigger of the right inferences when the occasion arises, (iv) a trigger of the right actions when occasion arises, and (v) its being held by a subject who prefers to believe what is true and gather further evidence when called for. A belief's being externally rational amounts to its being based on phenomenal experiences that are the result of properly functioning cognitive capacities or reliable belief-forming processes. (The internal/external rationality distinction drawn here is very much inspired by Plantinga 2000: 110-12. Note, though, that the external rationality part is more liberal.)

Internal rationality tracks whether a given belief is appropriately held given the subject's phenomenal experiences and mental states. It is tied to the subject's perspective - to factors that are internal to the subject. External rationality goes beyond the perspective of the subject. It tracks the epistemic status of the genesis or history of beliefs.

Consider (Pasta*), a case obtained by adding the following information about Joe to (Pasta):

(Pasta*) - Joe is justified in believing about himself that he is not drunk; is not under the influence of any drugs; has 20/20 vision; and that he has no history of hallucinating in the 
past. Furthermore, Joe does not have this kind of information about Jen. Jen is having veridical perceptual experiences. However, unbeknownst to him Joe has been drugged with a hallucinogen. He is in fact hallucinating Sarah's presence although he is not aware that he has been drugged and there are no way that he could discover it through experience, reflection, or introspection.

What is the rational response in the face of the disagreement? Let us consider external and internal rationality in turn.

Jen is externally rational while Joe is not. Her belief that Sarah is not present is based on phenomenal experiences that were brought about by a reliable belief-forming process. Recognizing the disagreement with Joe does nothing to undermine the good epistemic standing of the genealogy of Jen's belief. Joe's belief, on the other hand, is the result of hallucination. Hence, it fails to have the right epistemic pedigree and is not externally rational. ${ }^{4}$

Matters are different when it comes to internal rationality. Jen's belief is an appropriate response to her phenomenal experiences and mental states and integrates in the right way with inference and action. The same goes for Joe's belief. E.g., if he believes that Sarah wants to have wine with pasta, he will infer that Sarah wants to drink wine. Furthermore, if Joe always wants to accommodate Sarah's desire to drink wine, he will go fetch a bottle of wine upon forming the belief that Sarah wants to drink wine. For both Jen and Joe pressure to doxastically revise in order to maintain coherence in the face of the disagreement is alleviated by their respective symmetrybreakers (i.e. justified beliefs to the effect that their own epistemic situation is good and the absence of such beliefs about the other party).

These considerations on (Pasta*) suggest that verdicts on specific cases may well depend on what kind of rationality is taken to be relevant to the issue of the epistemic significance of recognized peer disagreement. How do the views considered above relate to the external/internal rationality distinction, and what verdict do they issue regarding (Pasta*)? 
Let us start with conformism. The unifying commitment of conformist views is that it is not rational to maintain one's initial doxastic attitude in the face of recognized peer disagreement. This is so on the equal weight view, the total evidence view, and any other conformist view. According to these views, once the disagreement comes within one's purview, doxastic revision is rationally required. Since this demand is driven by the internalization of the fact of disagreement, we take the primary focus of conformists to be internal rationality.

How about non-conformist views? Here the picture is more complex. Some non-conformists seem to have in mind external rationality, others internal rationality, and yet others a hybrid kind of rationality.

On the right reasons view the subject who in fact reasoned correctly can rationally maintain her initial doxastic attitude while the other party cannot. What matters is the genealogy of the beliefs of the disagreeing parties: who in fact reasoned correctly from the evidence. This very much suggests an external notion of rationality.

Non-conformists who are moved by the I'm-right-you're-wrong line of reasoning think that each party to the disagreement gets evidence that the other party is wrong upon learning of the disagreement. It is the internalization of the fact of disagreement and the accompanying epistemic 'downgrade' of the other party that underwrite the rationality of each party's maintaining their initial doxastic attitude. This points to rationality in the internal sense.

Non-conformists of the justificationist stripe operate with a notion of rationality that is sensitive to both external and internal factors. Recall that two things underwrite rational nonrevision on the justificationist view: a high degree of justified confidence and a symmetry-breaker. Jen and Joe both have symmetry-breakers, i.e. justified beliefs to the effect that their own epistemic situation is good and an absence of such beliefs about the other party. However, only Jen has a high degree of justified confidence. This is explained by the genesis of their respective beliefs which 
involves respectively veridical perception (Jen) and hallucination (Joe). Since Jen has both a high degree of justified confidence and a symmetry-breaker, she can rationally maintain her belief that Sarah is not present. Joe cannot rationally his conflicting belief because he fails to have a high degree of justified confidence. The rationality of Jen's belief is of a mixed kind. It is sensitive to both an external factor and an internal one. Since symmetry-breakers are constituted by beliefs, they are an internal determiner of rationality. However, the epistemic pedigree or history of beliefs is an external matter.

The reasoning as grounding view $(\mathrm{RGV})$ is a form of conformism and is thus concerned with internal rationality. Learning of a disagreement with a perceived peer defeats the wellgroundedness of each party's belief and thereby rationally mandates doxastic revision. However, RGV accommodates external rationality as well, at least after a fashion. The view grants that someone who has reasoned correctly to $p$ from evidence $E$ still has justification to believe $p$ (although, in the face of disagreement, they are not justified in believing $p$ ). In this sense it is still externally rational to believe $p$.

As just argued, different views on peer disagreement appear to operate with different notions of rationality. It is unfortunate that much of the peer disagreement debate-especially in its early stages - has unfolded somewhat in isolation from broader epistemological issues. For, differences concerning the epistemic significance of peer disagreement may well be interlinked with one's broader epistemological commitments - in particular, one's explicit or implicit commitments regarding the nature of rationality and justification. Hence, being clear about basic commitments concerning the nature of rationality could help clarify the peer disagreement debate.

Our own line on the issue concerning rationality is as follows. Most contributors to the debate implicitly assume that there is a single notion of rationality relevant to addressing the issue of the epistemic significance of recognized peer disagreement. We beg to differ. William Alston 
(2005) argued that there is a plurality of epistemic desiderata, and that it is futile to try to restrict the focus of epistemology to just one of them (see also Pedersen 2017). Inspired by this kind of pluralist attitude we suggest pluralism about epistemic rationality as an interesting, viable option. When it comes to framing the peer disagreement debate, we do not take internal rationality and external rationality to be mutually exclusive. Rather, they are complementary. Internal rationality and external rationality both constitute positive epistemic standings. They are both philosophically interesting species of rationality and, as such, both are relevant to the issue of the epistemic significance of recognized peer disagreement.

\section{References}

Alston, W. (2005). Beyond 'Justification': Dimensions of Epistemic Evaluation. Ithaca: Cornell University Press.

Ballantyne, N. and E. J. Coffman. (2011). Uniqueness, Evidence, and Rationality. Philosopher's Imprint, 11 (pp. 1-13).

Burge, T. (2010). The Origins of Objectivity. Oxford: Oxford University Press.

Christensen, D. (2007). Epistemology of disagreement: The good news. Philosophical Review, 116, pp. $187-217$.

—. (2008). Disagreement as evidence: The epistemology of controversy. Philosophy Compass, 4(5), pp. 756-767.

—. (2011) "Disagreement, Question-Begging and Epistemic Self-Criticism", Philosophers' Imprint, 11 (6): 1-22.

—. (2016). Conciliation, Uniqueness and Rational Toxicity. Noûs, 50, pp. 584-603. 
Christensen, D. \& J. Lackey. (Eds.) (2013). The Epistemology of Disagreement: New Essays. Oxford: Oxford University Press.

Elga, A. (2007). Reflection and disagreement. Nô̂s, 41, pp. 478-502.

Feldman, R. (2006). Epistemological puzzles about disagreement. In S. Hetherington (Ed.), Epistemology futures (pp. 216-236). Oxford: Clarendon Press.

—. (2007). Reasonable religious disagreements. In L. Anthony (Ed.), Philosophers without gods (pp. 194-214). Oxford: Oxford University Press.

Friedman, J. (2013). Suspended Judgment. Philosophical Studies, 162(2): 165-181.

Foley, R. (2001). Intellectual Trust in Oneself and Others. Cambridge: Cambridge University Press. Kelly, T. (2005). The epistemic significance of disagreement. In J. Hawthorne \& T. Gendler (Eds.), Oxford Studies in Epistemology Vol. 1 (pp. 167-196). Oxford: Oxford University Press.

—. (2010). Peer Disagreement and Higher-Order Evidence. In R. Feldman and T. Warfield (Eds.), Disagreement (pp. 111-174). Oxford: Oxford University Press.

Kopec, M. and M. Titelbaum. (2016). The Uniqueness Thesis. Philosophy Compass, 11 (pp. 189200).

Lackey, J. (2010a). A justificationist view of disagreement's epistemic significance. In A. Haddock, A. Millar, \& D. Pritchard (Eds.), Social Epistemology (pp. 298-325). Oxford: Oxford University Press.

—. (2010b). What should we do when we disagree? In J. Hawthorne \& T. Gendler (Eds.), Oxford Studies in Epistemology Vol. 3 (pp. 274-293). Oxford: Oxford University Press.

Lord, E. (2014). From Independence to Conciliationism: An Obituary. Australasian Journal of Philosophy, 92 (pp. 365-77).

Pedersen, N. J. L. L. (2017). Pure epistemic pluralism. In A. Coliva \& N. J. L. L. Pedersen (Eds.), Epistemic Pluralism (pp. 47-92). 
- (2018). Non-rational action in the face of disagreement: an argument against nonconformism. To appear in Synthese.

Plantinga, A. (2000). Warranted Christian Belief. New York: Oxford University Press.

Schoenfield, M. (2014). Permission to Believe: Why Permissivism Is True and What It Tells Us About Irrelevant Influences on Belief. Nô̂s, 48 (pp. 193-218).

Sidgwick, H. (1907). The Methods of Ethics. London: Palgrave.

Sosa, Ernest (2010). "The Epistemology of Disagreement", in A. Haddock, A. Millar and D. Pritchard (Eds), Social Epistemology (pp. 278-297). Oxford: Oxford University Press.

Weatherson, B. (2013). Disagreements, Philosophical and Otherwise. In Christensen and Lackey 2013 (pp. 54-73).

Wedgwood, R. (2007). The Nature of Rationality. Oxford: Oxford University Press.

White, R. (2005). Epistemic Permissiveness. Philosophical Perspectives, 19 (pp. 445-459).

Wietmarschen, H. van. (2013). Peer Disagreement, Evidence, and Well-Groundedness. Philosophical Review, 122, (pp. 395-425).

\section{Further reading:}

Cohen, S. (2013). A Defense of the (Almost) Equal Weight View. In Christensen and Lackey 2013 (pp. 98-117).

Enoch, D. (2010). Not Just a Truthometer: Taking Oneself Seriously (but not Too Seriously) in Cases of Peer Disagreement. Mind, 119 (pp. 953-97).

Feldman, R. Evidentialism, Higher-Order Evidence, and Disagreement. Episteme, 6 (pp. 294-312). Hazlett, A. (2013). Entitlement and Mutually Recognized Reasonable Disagreement. Episteme, 11 (pp. 1-25). 
Kelly, T. (2013). Disagreement and the Burdens of Judgment. In Christensen and Lackey 2013 (pp. $31-53)$.

Kopec, M. and M. Titelbaum. (Forthcoming). When Rational Reasoners Reason Differently. To appear in M. Balcerak-Jackson and B. Balcerak-Jackson (Eds.), Reasoning: Essays on Theoretical and Practical Thinking. Oxford: Oxford University Press.

Lackey, J. (2013). Disagreement and Belief Dependence: Why Numbers Matter. In Christensen and Lackey 2013 (pp. 243-68).

Titelbaum, M. (2015). Rationality’s Fixed Point (Or: In Defense of Right Reason). T. Gendler and J. Hawthorne (Eds.), Oxford Studies in Epistemology Vol. 5 (pp. 253-94).

Wilson, A. (2010). Disagreement, Equal Weight and Commutativity. Philosophical Studies, 149, (pp. 321-26).

\footnotetext{
${ }^{1}$ Nikolaj J. L. L. Pedersen gratefully acknowledges support from the National Research Foundation of Korea (grants no. 2013S1A2A2035514 and 2016S1A2A2911800). Filippo Ferrari would like to acknowledge the support of the German Research Foundation (DFG-BR 1978/3-1, 'Disagreement in Philosophy').

${ }^{2}$ (Significance-Peer) is often qualified and framed in terms of (at least) approximate epistemic peerhood, as it is very rare that people regard each other as exact peers.

${ }^{3}$ Foley $(2001: 108,110,114)$, Kelly $(2005: 179-180)$. Another argument from epistemic egocentrism can be found in Wedgwood (2007: 261).

${ }^{4}$ In saying this we are assuming that the kind of proper functioning or reliability relevant to external rationality is incompatible with hallucination. This is Plantinga's view and shared by many others, including Burge 2010 .
} 